\title{
Articulation rate in adverse listening conditions in younger and older adults.
}

\author{
Outi Tuomainen ${ }^{1}$, Valerie Hazan ${ }^{1}$ \\ ${ }^{1}$ Speech Hearing and Phonetic Sciences, UCL, UK \\ o.tuomainen@ucl.ac.uk, v.hazan@ucl.ac.uk
}

\begin{abstract}
Speech communication becomes increasingly difficult with age, especially in adverse listening conditions. We compared speech adaptations made by 'older adult' (65-84 years) and 'younger adult' (19-26 years) talkers when speech is produced with communicative intent. The aim was to investigate how articulation rate is affected by the type of adverse listening condition and by the change in task demands. Articulation rate was recorded in 35 older and 18 younger adult talkers when they were reading and repeating BKB-sentences and when they were doing an interactive 'spot-the-difference' game in a good and three adverse listening conditions (Hearing Loss Simulation, one speaker in noise, both speakers in noise). Similar to younger adults, older adults reduced their articulation rate in the cognitively simpler sentence repetition task in response to adverse conditions. However, in spontaneous speech, only older adult women decreased their articulation rate to counter the effect of the adverse conditions to the same degree as the younger adult talkers. Older men did not reduce their articulation rate in any of the three adverse conditions. These sex differences were not due to differences in the task difficulty experienced by men and women nor were they associated with sensory or cognitive factors.
\end{abstract}

Index Terms: aging, speech production, clear speech strategies, adverse listening conditions, articulation rate.

\section{Introduction}

Several physiological, sensory and cognitive factors can interact and affect the speech production abilities of older adult (OA) speakers. For example, it has been shown that with advancing age, laryngeal and respiratory function can change resulting in altered voice characteristics and in decreased utterance length [1]. Furthermore, in normal aging, auditory acuity decreases especially at higher frequency ranges, and there are also changes in attentional capacity and memory function $[2,3]$. Together these age-related changes can lead to general slowing of sensory and motor performance, reduced motor control and sensory feedback that can have adverse effects for the entire speech production system.

Previous studies on age effects in speech production have reported an overall reduction of speech rate (i.e., fewer syllables per second) in OA speakers. In a study by Smith et al., [4] older adults, between 66-75 years of age, produced longer segments, syllables and sentences when they were asked to repeat monosyllabic words and simple and complex sentences at their normal speaking rates and at fast speaking rates. Overall, OA talkers were $20-25 \%$ slower than younger adult (YA) talkers both at their normal speaking rates and at fast rates, and were also $55 \%$ more variable than YA talkers. In a more recent study, Gooze and colleagues [5] assessed the lingual kinematic strategies used by YA and OA talkers to increase speaking rate. They measured tongue movement with electromagnetic articulography for repetition of $/ \mathrm{ta} / \mathrm{and} / \mathrm{ka} /$ syllables at moderate and fast speaking rates. They reported that both YA and OA talkers used similar strategies to increase the speaking rate. However, OA talkers showed overall slower syllable repetition rates, decreased tongue velocity and acceleration values compared to YA talkers. They suggested that these differences could be due to age-related physiological, neuromotor and sensory declines.

However, speech communication often takes place in unfavorable conditions where speakers must adjust their speech in order to maintain good communication. It is well established that YA talkers are skilled in adapting their speech production in ways that counter the effects of the communication barrier by adopting what is called a 'clear speaking style' $[6,7]$. Moreover YA talkers can tailor the acoustic-phonetic aspects of their speech to match the particular adverse condition [8]. OA talkers, in turn, often report having particular difficulty communicating in challenging listening conditions, e.g. in noise or in the presence of other talkers. To date, most studies on speech production and aging have focused on analyzing controlled materials such as reading or repeating syllables, words or sentences in optimal listening conditions. Much less is known about the effects of aging on speech that is produced in ecologically valid communicative situations that have higher cognitive load than a word or sentence repetition task, and under both good and adverse listening conditions.

The present study set out to examine the degree to which YA and OA talkers adapt their speech to counter the effect of adverse listening conditions when speech is produced with communicative intent. Whilst talkers can adjust several parameters of their speech in an attempt to maximize communicative efficiency, here we focused on articulation rate which has been shown to be a prominent strategy when talkers attempt to clarify their speech [9]. In this study, we elicited speech using two types of tasks that differ in cognitive demands: (i) read BKB sentences [10], produced within an interactive task and (ii) spontaneous speech produced in a cooperative 'spot the difference' picture task (the DiapixUK task; [11]). These two tasks produce sentences that are similar in complexity, but reading sentences requires less planning and cognitive resources than producing spontaneous speech within a problemsolving task [12]. In order to see whether older and younger talkers slowed their articulation rate as a 'clear speech strategy' when communication became effortful, the tasks were run both in good and adverse conditions. In order to see whether the type of adverse condition had an effect on the degree of articulation rate decrease, three different types of adverse listening conditions that differ in the type of degradation they entail were used: YA and OA talkers were communicating with a YA interlocutor who (i) had a simulated severe-to-profound hearing 
loss; (ii) was in multi-talker babble, and (iii) when both talkers were in multitalker babble. In the first two conditions the speech adaptations are purely made for the benefit of the interlocutor whereas in the final condition both talkers are directly affected by the adverse condition. In the three adverse conditions, speech adaptations are naturally elicited by introducing a communication barrier leading the talker to have to clarify her/his speech to maintain effective communication with the interlocutor.

We predicted that, in-line with previous research, OA talkers would be slower speakers than YA talkers. Furthermore, because previous research has shown women to be both slower talkers and readers than men, we expected women to have a slower articulation rate than men in both good and adverse listening conditions and in both tasks [13, 14]. For task differences, we predicted that all talkers would be better able to adapt their speech in challenging listening conditions in the cognitively less demanding sentence reading task. However, in the spontaneous speech task that requires more planning and has a higher cognitive load than the sentence reading task, we predicted that OA talkers would be less able to adapt their speech in the adverse conditions than younger adults [15]. We also expected that the OA talkers would be particularly affected when directly affected by the communication barrier. Lastly, we expected to see a relationship between sensory acuity, cognitive function and speech adaptations. We expected that individuals with better sensory and/or cognitive ability would be more skilled speakers and more efficient communicators than individuals with mild hearing loss and/or lower working memory capacity.

\section{Method}

\subsection{Participants}

Speech was recorded from 53 single-sex pairs of native Southern English adult talkers between the ages of 18 and 85 . The pairs did not know each other and had not met each other before the recording. Each participant was assigned a role of a primary talker ('Talker A') or a secondary talker ('Talker B'). Primary talkers were divided into two age groups: 'younger adults' (YA) between 19-26 years of age (9 F, $9 \mathrm{M})$ and 'older adults' (OA) between 65-84 years of age (23 F, $12 \mathrm{M}$ ). Secondary talkers were always younger adults $(\mathrm{N}=53$, between 18-30 years of age) of the same sex as the Talker A. Participants reported no history of speech or language impairments. YA participants passed a hearing screen at $25 \mathrm{~dB} \mathrm{HL}$ or better at octave frequencies between $250-8000 \mathrm{~Hz}$ in both ears. OA participants had either 'normal hearing' $(\mathrm{N}=23 ; 8$ Male) defined as a hearing threshold of $<25 \mathrm{~dB}$ between octave frequencies $250-4000 \mathrm{~Hz}$ or 'mild hearing loss' $(\mathrm{N}=12$; 4 Male) defined as a hearing threshold of $<45 \mathrm{~dB}$ in this frequency range with a symmetrical downward slope of pure tone threshold in the highfrequency range typical for an age-related hearing loss profile.

\subsection{Procedure}

The BKB sentence repetition and the Diapix tasks were carried out in four transmission conditions: "normal transmission' (NORM), Talker B in 'hearing loss simulation' (HLS), Talker B in multitalker babble (BAB-1), and both talkers in multitalker babble (BAB-2).

In HLS, Talker B heard Talker A via a real-time hearing loss simulator modelling a profound sensorineural loss at levels
40-50-60-90-90 dB at frequencies 250-500-1000-4000-8000; (HELPS, [16]). In BAB-1 condition, Talker B heard Talker A in 8-talker babble noise that was similar in degree of communicative difficulty to the HLS condition. The SNR for the BAB-1 condition was individually set using an adaptive procedure to equate HLS performance on the Modified Rhyme Test (MRT). In BAB-2 condition both speakers communicated in the same 8-talker babble noise with a $0 \mathrm{~dB}$ SNR across all speakers. During the recordings the two participants sat in different rooms and communicated via Eagle G157b lapel microphones. They were unable to see each other so they could only use auditory information to complete the tasks. The speech of each participant was recorded on a separate channel at a sampling rate of $44100 \mathrm{~Hz}$ (16 bit) using an EMU 0404 USB audio interface and Adobe Audition and Rode NT1-A condenser microphones.

In the BKB task, a subset $(\mathrm{N}=128)$ of the $\mathrm{BKB}$ sentences were used ( 32 sentences/condition). The BKB sentences are meaningful sentences with a simple syntactic structure and lexicon. In this task, Talker A read out each sentence one by one and Talker B were asked to repeat the sentence verbatim. They were told that their performance will be scored but Talker A was not allowed to repeat the sentence or correct Talker B. Therefore, in order to achieve a high score, Talker A had to adopt a clear speaking style in the three adverse listening conditions. In the Diapix task, using the Diapix picture pairs to elicit spontaneous speech, the two participants played an interactive 'spot the difference' game [11]. In this task, each participant was given a different version of the same picturescene (version A and B, see Figure 1), and were told the pictures contained 12 differences which they had to find. Talker A was leading the conversation and was instructed to do most of the talking, whereas Talker B was mainly required to ask questions and make suggestions. They were given 10 minutes to find these differences. In both the BKB and Diapix tasks, the participants always started with the NORM condition and the order of the three adverse conditions was randomized within age groups.

In order to investigate the relationship between sensory and cognitive function and speech adaptations, we measured hearing threshold levels with pure tone audiometry (PTA) and working memory capacity. For sensory function, we used the better ear average between octave frequencies $250-4000 \mathrm{~Hz}$. For working memory we used the backward digit span (DSB) test that measures information storage and rehearsal [17]. In the DSB task, the participant repeated auditory presented number sequences in a reverse order (e.g., for the sequence 2-5-7 they had to say 7-5-2) and they were scored correct/incorrect for each sequence (maximum score $=14$ ).

\subsection{Data processing}

For all recordings, each channel was automatically transcribed using cloud-based speech recognition system by Speechmatics (https://www.speechmatics.com/). An in-house Matlab script was used to upload and download files to the Speechmatics database and create a Praat textgrid from the original JSON format. These automated transcriptions and the audio-transcription alignment were then hand-checked at a word level and corrected for errors.

Articulation rate was calculated as the number of syllables produced by Talker A divided by the total duration of speech regions for that talker. Syllable counts were calculated from the orthographic transcriptions of the spontaneous speech using the qdap package in $\mathrm{R}$ [18], after exclusion of segments labelled as 
unfinished words, hesitations, fillers and agreements (e.g. 'yeah', 'yup', 'err', 'hmm').

Street 3

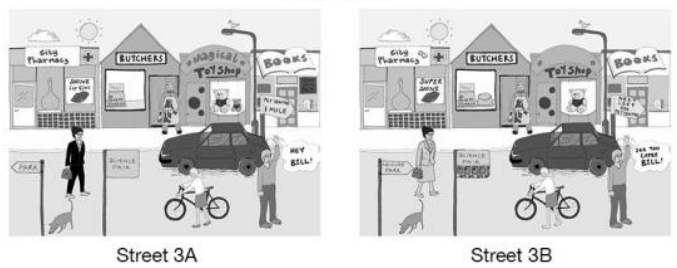

Figure 1. A picture pair from the DiapixUK picture set.

\section{Results}

A repeated measures anova was run with the four listening conditions (NORM, HLS, BAB-1, BAB-2) as within-subjects factors and Age (YA, OA) and Sex (M,F) as between-subjects factors separately for the BKB sentence reading and Diapix tasks. A Bonferroni adjustment was applied to all follow-up analyses. To highlight the amount of change in articulation rate between conditions, in Figures 2-3, articulation rate is represented as a percentage change relative to the condition where the two talkers could hear each other normally:

$(($ Adverse condition - NORM) / NORM $) * 100$

All analyses were done on the speech of the primary talkers (Talker A) because they had to overcome the adverse condition affecting their interlocutor.

\subsection{BKB sentence reading}

There was a significant main effect of Condition $(F(3,114)=39.085, p<.001)$ : when repeating sentences, all participants reduced their articulation rate in the three adverse conditions when compared against the NORM condition (see Figure 2; all comparisons $\mathrm{p}<.003$ ). The articulation rate was $25 \%$ slower for the HLS relative to NORM and $20 \%$ and $8 \%$ slower for the BAB-1 and BAB-2 conditions re NORM, respectively. The three adverse conditions were significantly different from each other $(\mathrm{p}<.003)$ with rate reducing from NORM (3.36), BAB-2 (3.07), BAB-1 (2.73) to HLS (2.53 syllables/second).

There was a significant main effect of Age $(F(1,38)=5.554$, $\mathrm{p}=.024)$ : YA talkers were faster speakers than OA talkers (3.06 vs. 2.79 syllables/second). No main effect or interactions with factor Sex were found $(\mathrm{p}>.1)$.

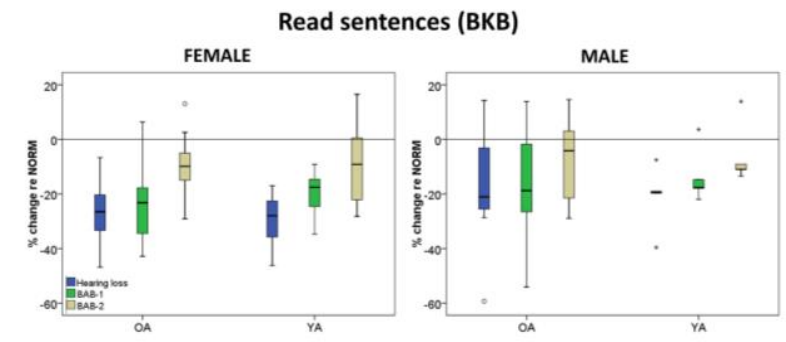

Figure 2. Percentage relative change (re NORM) in articulation rate in the three adverse conditions (HLS, BAB-1, $B A B-2)$ in older $(n=29)$ and younger adults $(n=13)$.

\subsection{Spontaneous speech (DiapixUK)}

There were significant main effects of Condition $(F(3,123)=14.329, \mathrm{p}<.001)$ and $\operatorname{Sex}(\mathrm{F}(1,41)=14.886, \mathrm{p}<.001)$, and a significant interaction between Condition, Age and Sex $(\mathrm{F}(3,123)=2.904, \quad \mathrm{p}=.038)$. The main effect of Age $(\mathrm{F}(1,41)=3.300, \mathrm{p}=.077)$ and interaction between Condition and Age were approaching significance $(\mathrm{F}(3,123)=2.318, \mathrm{p}=.079)$.

Overall, all participants reduced their articulation rate in spontaneous speech (NORM) by $5 \%$ for HLS $(\mathrm{p}<.001)$, and by $4 \%$ for BAB-1 which was approaching significance $(p=.062)$ but only by and $2 \%$ for BAB-2 ( $\mathrm{p}=.186)$. Articulation rate for HLS was significantly lower than in all other conditions $(\mathrm{p}<.001)$. The difference between BAB-1 and BAB-2 was not significant $(p>9)$. Furthermore, female talkers had a lower articulation rate than male talkers $(3.50$ vs. 3.88 syllables/second) and OA talkers had a marginally lower articulation rate than YA talkers $(3.60$ vs. 3.78 syllables/second).

\section{Spontaneous speech (DiapixUK)}
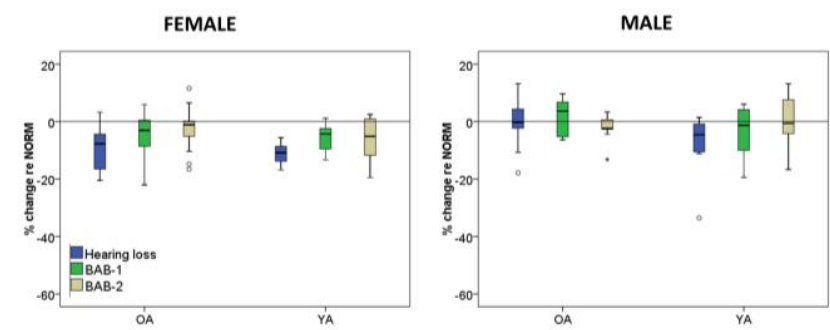

Figure 3. Percentage relative change (re NORM) in articulation rate in the three adverse conditions (HLS, BAB-1, $B A B-2)$ in older $(n=29)$ and younger adults $(n=17)$.

The three-way interaction between Condition, Age and Sex revealed a different profile for female and male talkers (see Figure 2). All female talkers significantly reduced their articulation rate for the three adverse conditions $(\mathrm{F}(3,78)=18.572, \mathrm{p}<.001 ; \mathrm{NORM}$ versus HLS, $\mathrm{p}<.001$; NORM versus $\mathrm{BAB}-1$ and $\mathrm{BAB}-2$, both comparisons $\mathrm{p}<.05$, BAB- 1 versus $B A B-2, p>$.9). On average they reduced their articulation rate by $6 \%$ for HLS and by $5 \%$ and $2 \%$ for BAB- 1 and BAB2 , respectively. Furthermore, the YA female talkers were overall faster talkers than female OA talkers $(\mathrm{F}(1,26)=5.987$, $\mathrm{p}=.021$ ).

For male talkers, however, none of the main effects were significant $(\mathrm{p}>\mathrm{.1})$ but the interaction between Condition and Age was significant $(F(3,45)=3.136, p=.035)$. This interaction revealed that significant articulation rate reduction for adverse conditions was only achieved by the YA male talkers and for the HLS condition only $(\mathrm{p}=.048$; other comparisons $\mathrm{p}>.1)$. The YA male talkers reduced their articulation rate to HLS by $9 \%$ whereas OA male talkers decreased their articulation rate to HLS by only $1 \%$. In the diapix task, OA male talkers did not reduce their articulation rate to any of the three adverse conditions (see Figure 3).

In sum, the results from the sentence reading task indicate that in a cognitively simple task such as sentence repetition, older adults are slower speakers than younger adults. Furthermore, older adults make similar adaptations to younger adults in their articulation rate when communicating in challenging conditions. However, the results from the 
spontaneous speech task indicate that older adults are (marginally) slower speakers than younger adults. Furthermore, our results show that older women adapt their articulation rate when communicating in adverse conditions in a similar way to younger women. However, both male groups (YA and OA) reduced their articulation rate less than female talkers. In fact, older men did not adjust their articulation rate to any of the adverse conditions.

\subsection{Effects of sensory and cognitive factors and task difficulty}

Average hearing thresholds and working memory spans for both age groups and men and women separately are presented in Table 1. Pearson's correlation between percentage change in articulation rate re NORM condition (HLS, BAB-1, BAB-2), hearing thresholds and working memory span separately for YA and $\mathrm{OA}$ age groups revealed no significant associations between any of these variables ( $>>055$; Bonferroni corrected significance level $p=.013$ ) in either sentence reading or spontaneous speech tasks. This indicates that, in these tasks, talkers with better hearing or cognitive function (as evidenced by a working memory span) were not more skillful at adapting their speech in adverse conditions. However, it is possible that male talkers simply found the task easier and thus needed to adapt their speech less in the adverse conditions. Therefore, as a measure of experienced task difficulty, we compared the number of differences (out of 12) found in 10 minutes between age groups and sex. The results showed a main effect of Condition $(F(3,126)=5.920, \quad p=.001)$ : participants found significantly less differences in 10 minutes in HLS than in NORM and BAB-2 $(p<.022)$ but not in BAB-1 $(p=.268$; all other comparisons $p>9$ ). However, no significant main effects or interactions involving Age or Sex were found (YA-female: 11.6; YA-male: 11.3; OA-female: 10.5; OA-male: 11.1 differences; $p>.2$ for all comparisons). This suggests that the fact that men, and older adult men in particular, reduced their articulation rate less than women in adverse condition is not because they found the task easier and did not need to adopt a clear speaking style.

Table 1. Hearing thresholds (Better ear average at 250$4000 \mathrm{~Hz}$ ) and Digit Span Backwards (DSB) scores in younger (YA) and older (OA) adults (standard deviations in brackets).

\begin{tabular}{|l|l|l|l|l|}
\hline \multirow{2}{*}{} & \multicolumn{2}{|l|}{ YA } & OA \\
\cline { 2 - 5 } & F & M & F & M \\
\hline PTA & 3.88 & 0.60 & 20.09 & 23.70 \\
& $(3.23)$ & $(0.55)$ & $(10.51)$ & $(6.98)$ \\
\hline DSB & 6.88 & 8.50 & 7.89 & 7.30 \\
& $(1.81)$ & $(3.94)$ & $(1.45)$ & $(2.06)$ \\
\hline
\end{tabular}

\section{Discussion}

The aim of this study was to investigate the effects of aging in clear speech adaptations in ecologically-valid challenging communicative environments. We compared articulation rate in younger and older adults in three different adverse listening conditions and in two different tasks that differ in their cognitive demands. As predicted, older adults were slower speakers than younger adults in sentence reading and marginally so also in the spontaneous speech task. Furthermore, women were slower speakers than men but only in the spontaneous speech task. For speech adaptations, our results showed that older adults are able to adjust their articulation rate as a clear speech strategy in a similar way to younger adults to counter the effect of communication barrier in a simple sentence reading task. In this task, all talkers significantly reduced their articulation rate for all three adverse conditions. In cognitively more challenging spontaneous speech task, older adult female talkers slowed down their speech to the same degree as younger female talkers in all adverse conditions. The younger and older adult male talkers, however, reduced their articulation rate less than female talkers. In fact, older adult male talkers made no adjustments to their speech for the benefit of the other talker or when they were directly affected by the communication barrier whereas younger male talkers slowed down their speech when their interlocutor had a simulated severe-to-profound hearing loss. These differences between YA and OA male talkers were not due to either sensory (hearing level) or cognitive (working memory) factors or due to task difficulty. In a meta-analysis of sex differences in interaction styles, Wood [19] reported that, when given a collaborative task, men are more likely to adopt more authoritarian techniques than women. Thus, the differences between men and women observed in the current study could be attributable to social rather than sensory/cognitive factors. Lastly, against our predictions, our results showed that OA talkers were not less able than YA talkers to adapt their speech to counter the effect of a communication barrier when they directly experienced it themselves (BAB-2). When talking in multitalker babble all OA talkers, however, reported that they found the task challenging. Therefore, it is possible that articulation rate is a fairly robust clear speech strategy and there are age-related changes in other acoustic-phonetic features or voice characteristics that are involved in communicating in challenging listening conditions (e.g., pitch, loudness or segmental enhancements of speech sounds). It would also be important to look at individual strategies when the primary talker is in noise because some older adult talkers may choose to speed up instead of slowing down their speech in response to background noise in order to minimize the task duration.

\section{Conclusions}

When speech is produced with communicative intent, older adults are able to adjust their speech to maintain effective communication in a cognitively less demanding task. However, in a task that requires more cognitive resources older adult men did not clarify their speech when communication became effortful. This was not explained by sensory or cognitive factors or by task difficulty.

\section{Acknowledgements}

This work was supported by the Economic and Social Research Council [grant number: ES/L007002/1]. We acknowledge the contribution of our collaborators Doug Brungart, Benjamin Sheffield (Walter Reed National Military Medical Center) and Joseph Desloge (Sensimetrics Corporation) to the design of the HLS condition, and of our collaborators Jeesun Kim and Chris Davis (MARCS Institute) to the design of the study. We thank all volunteers who took part in the study. 


\section{References}

[1] J. E. Huber and J. Spruill, "Age-related changes to speech breathing with increased vocal loudness", Journal of Speech, Language, and Hearing Research, vol 51, pp. 651-668, 2008.

[2] K. M. Wood, J. D. Edwards, O. J. Clay, V. G. Wadley, D. L. Roenker and K. K. Ball, "Sensory and cognitive factors influencing functional ability in older adults", Gerontology, vol 51, pp. 131-41, 2005.

[3] T.A. Salthouse, T.M. Atkinson and D.E. Berish. "Executive function as a potential mediator of age-related cognitive decline in normal adults", Journal of Experimental Psychology: General, vol. 132, pp. 566-594, 2003.

[4] B. L. Smith, J. Wasowicz, and J. Preston, "Temporal Characteristics of the Speech of Normal Elderly Adults", Journal of Speech and Hearing Research, vol. 30, pp. 522-529, 1987.

[5] J. V. Goozee, D. K. Stephenson, B. E. Murdoch, R. E., Darnell, and L. L. Lapointe, "Lingual kinematic strategies used to increase speech rate: comparison between younger and older adults", Clinical Linguistics and Phonetics, vol. 19, pp. 319 334, 2005.

[6] R. Smiljanic, R. and A. R. Bradlow, "Speaking and Hearing Clearly: Talker and Listener Factors in Speaking Style Changes", Language and Linguistics Compass, vol. 3, pp. 236264, 2009.

[7] V. Hazan, and R. Baker, "Acoustic-phonetic characteristics of speech produced with communicative intent to counter adverse listening conditions", The Journal of the Acoustical Society of America, vol. 130, pp. 2139-2152, 2011.

[8] M. Cooke, and Y. Lu, "Spectral and temporal changes to speech produced in the presence of energetic and informational maskers", The Journal of the Acoustical Society of America, 128, pp. 2059-2069, 2010

[9] V. Hazan, O. Tuomainen, and M. Pettinato. "Suprasegmental Characteristics of Spontaneous Speech produced in Good and Challenging Communicative Conditions by Talkers aged 9 to 14 years old", Journal of Speech, Language, and Hearing Research, In Press, 2016.

[10] J. Bench, Å. Kowal and J. Bamford, "The BKB (BamfordKowal-Bench) sentence lists for partially-hearing children", British. Journal of Audiology, vol. 13, pp. 108-112, 1979.

[11] R. Baker, and V. Hazan, "DiapixUK: task materials for the elicitation of multiple spontaneous speech dialogs", Behavior Research Methods, vol. 43, pp. 761-770, 2011.

[12] V.M. Holmes, "Hesitations and sentence planning", Language and Cognitive Processes, vol. 3, pp. 323-361, 1988.

[13] D. Byrd, "Preliminary results on speaker-dependent variation in the TIMIT database", Journal of the Acoustical Society of America, vol. 92, pp. 593-596, 1992.

[14] S. P. Whiteside, "Temporal-based speaker sex differences in read speech: A sociophonetic approach", In Proceedings of the XIIIth International Congress of Phonetic Sciences (ICPhS 95), vol. 3, pp. 516-519, 1995.

[15] R. Smiljanic, "Can older adults enhance the intelligibility of their speech?", JASA Express Letters, vol. 133, pp. 129-135, 2013

[16] P. M. Zurek, and J. G. Desloge, "Hearing loss and prosthesis simulation in audiology", Hearing Journal, vol. 60, pp 3233,36,38. 2007.

[17] A. Baddeley, "Working Memory and Language: An overview", Journal of Communication Disorders, vol. 36, pp. 189-208, 2003.

[18] T. W. Rinker, qdap: Quantitative Discourse Analysis Package, University at Buffalo/SUNY, Buffalo, New York, URL http://github.com/trinker/qdap, (version 2.2.0. 125). 2013.

[19] W. Wood. "Meta-analytic review of sex differences in group performance", Psychological Bulletin. vol. 102, pp. 53-71, 1987 